\title{
INSTRUMENTALE SPIELFORMELN UND VOKALE VERZIERUNGEN IM 16.JAHRHUNDERT
}

Theodor GöLLNER

\begin{abstract}
In the music of the 16th century, stereotype four-note formulas are found in the instrumental as well as in the vocal idiom. Although frequently identical in appearance, their functions are very different. While indispensable in the instrumental idiom, particularly in keyboard music, where they represent the very essence of instruction in 15th-century organ treatises and fundamenta, they appear in vocal music only as additions to finished compositions or existing practices. What amounts to an absolute necessity in one case is merely an ornamental supplement in the other. In vocal music it is treated as a matter of performance carried out by a qualified soloist and limited to the musical centers of Italy. The present article raises the question as to how the genuinely instrumental and widely spread keyboard practice made its impact on the vocal ornaments of the singer, whose primary concern is the musical communication of language.
\end{abstract}

\begin{abstract}
Abstrakt
Die in der Musik des 16. Jahrhunderts im Instrumentalen wie im Vokalen vorkommenden, meist viertönigen, stereotypen Formeln sind zwar in beiden Bereichen weitgehend identisch, doch von höchst unterschiedlicher Funktion. Während sie im Instrumentalen, besonders in der Tastenmusik, unverzichtbar sind und schon im 15 . Jahrhundert den Kern der Orgeltraktate und Fundamenta bilden, treten sie im Vokalen zu einer fertigen Komposition oder bestehenden Praxis hinzu. Was im einen Fall eine unbedingte Notwendigkeit ist, wird im anderen zu einem nachträglichen Ornament, das sich oft erst bei der Aufführung einstellt, den qualifizierten Solosänger voraussetzt und auf die musikalischen Zentren Italiens konzentriert war. Der Artikel versucht $\mathrm{zu}$ klären, wie sich das genuin instrumentale, allgemein verbreitete Tastenspiel auf die spezielle Verzierungskunst des Sängers auswirkt, dessen primäre Aufgabe es ist, Sprache musikalisch zu vermitteln.
\end{abstract}

Die Initiatoren der Florentiner Camerata mussten sich in ihrer Rückbesinnung auf die Musik der griechischen Antike vor allem der Vorherrschaft des Kontrapunkts erwehren. Das erstrebte Ziel einer mehr dem gesprochenen als dem gesungenen Wort verpflichteten Musik stiess aber noch auf einen anderen Widerstand, der aktueller war als der etablierte Kontrapunkt und deshalb schwerer zu überwinden schien: Die sprachnahe Rezitation sah sich bedroht von einer bewunderten Verzierungskunst, mit der sich die Sänger in den musikalischen Zentren Italiens hervortaten.

Nicht nur gegen den Kontrapunkt, sondern auch gegen die ausufernden "Passaggi" wendet sich deshalb Giulio Caccini in seiner Vorrede zu "Le nuove musiche" (Florenz 1601), wenn er "der schon früher gebräuchlichen Art von Passaggien entfliehen will, die mehr für Blas- 
und Saiteninstrumente als für die Stimme geeignet waren". ${ }^{1}$ Demnach war im Gegensatz zur Neuigkeit des stile recitativo das Passaggieren vor allem beim solistischen Vortrag von Vokalkompositionen bekannt, zumal wenn nur eine Stimme gesungen, die übrigen gespielt wurden. Da notenschriftliche Belege hierfür in größerer Zahl erst im letzten Jahrzehnt des 16. Jahrhunderts auftreten, wird die Praxis um diese Zeit in ein akutes Stadium getreten sein, dem eine Phase improvisierter Verzierungen vorausging. ${ }^{2}$ Rückschlüsse, die sich von der späteren Zeit ziehen lassen, legen nahe, dass vokale Verzierungen sowohl in der weltichen als auch in der geistlichen Musik üblich waren, vor allem in Madrigalen und Chansons bzw. Motetten, Psalmen und Magnificats. ${ }^{3}$

Anders verhält es sich im instrumentalen Bereich, in dem wir wesentlich früher als in den vokalen Diminutionslehren über die Spielpraxis insgesamt unterrichtet werden. Während in der Vokalkomposition die Diminutionsanweisungen das fertige Werk voraussetzen und dieses im Hinblick auf die Aufführung ergänzen, findet eine Trennung von Komposition und Aufführung im instrumentalen Bereich, speziell in der Tastenmusik, nicht statt. Diese wird schon in den Fundamenta und Orgeltraktaten des 15. Jahrhunderts stets als Ganzes behandelt, in dem sich Kompositorisches mit Spieltechnischem verbindet. ${ }^{4} \mathrm{Um}$ mehrstimmige Musik zu machen, müssen Konsonanzregeln befolgt und Spielverläufe erlernt, aber nicht fertige Kompositionen nachträglich verziert werden. Es gehört zu den Charakteristika des Orgelspiels, Zusammenklänge durch lineare Abläufe in stereotypen Formeln zu überbrücken. Die Gliederung erfolgt in der Regel nach konstanten Mensureinheiten, den Tactus, deren richtige Handhabung im Mittelpunkt der Lehre steht. Es ist bezeichnend, dass die Verbindungsformeln nicht als verzierende Diminutionen, sondern als elementare Bausteine in Form von Tactus angesehen und behandelt wurden. Der Begriff Diminution kommt deshalb im Umfeld des frühen Orgelspiels überhaupt nicht vor. Der Organist unterteilt und verkleinert nicht, sondern geht von kompakten Notengruppen aus, die zwar vervielfältigt und variiert, aber nicht auf einen übergeorneten Wert bezogen werden.

Der instrumentale Formelbestand bleibt im wesentlichen auch im 16. Jahrhundert erhalten. Wurde er zuerst in Deutschland im 15. Jahrhundert systematisch erfasst, so begegnet er in praktischen Quellen etwa gleichzeitig in Italien im Faenza-Codex ${ }^{5}$ und danach in den ersten

1. “...per isfuggire quella antica maniera di passaggi che già si costumarono, più propria per gli strumenti di fiato e di corde, che per le voci”. Hugo Goldschmidt, Die italienische Gesangsmethode des XVII. Jahrhunderts, Breslau 1892, Nachdruck Leipzig 1978, S. 14.

2. Ernst Ferand, Die Improvisation in der Musik, Zürich 1938, S. 261-269. Howard Mayer Brown, Embellishing Sixteenth-Century Music, London 1976, S. 65.

3. Ernest T. Ferand, "Didactic Embellishment Literature in the Late Renaissanse: A Survey of Sources", in: Aspects of Medieval and Renaissance Music. A Birthday Offering to Gustave Reese. Edited by Jan LaRue, New York 1978, S. 154172.

4. Theodor Göllner, Formen früher Mehrstimmigkeit, Tutzing 1961, S. 61-101. Willi Apel, Geschichte der Orgelund Klaviermusik bis 1700, Kassel usw. 1967, S. 42-52. Christian Meyer, "Ein deutscher Orgeltraktat vom Anfang des 15. Jahrhunderts", in: Musik in Bayern, 29, 1984, S. 43-59. Klaus Aringer, Die Tradition des Pausa- und Finale Schlusses in den Klavier- und Orgelwerken von Johann Sebastian Bach, Tutzing 1999, S. 21-86. Claus Bockmaier, Der Takt als musikalische Gestalt, Habilitationsschrift München 1997, Druck in Vorbereitung in: "Münchner Veröffentlichungen zur Musikgeschichte". 5. Michael Kugler, Die Tastenmusik im Codex Faenza, Tutzing 1972. Dragan Plamenac (Ed.), Keyboard Music of the Late Middle Ages in Codex Faenza 117. CMM 57, American Institute of Musicology 1972. 
gedruckten Quellen für Tastenmusik in Ricercaren und Intabulierungen vokaler Kompositionen. ${ }^{6}$ Ein kurzer Abschnitt aus dem "Recercare primo" von Marco Antonio Cavazzoni (Venedig 1523) zeigt eine bewegte, zwischen Vierteln und Achteln wechselnde Oberstimme über einem tragenden Unterbau, der die Gerüstklänge $D-A-E-D-E-D-G$ im Taktabstand setzt: ${ }^{7}$

\section{Beispiel 1:}
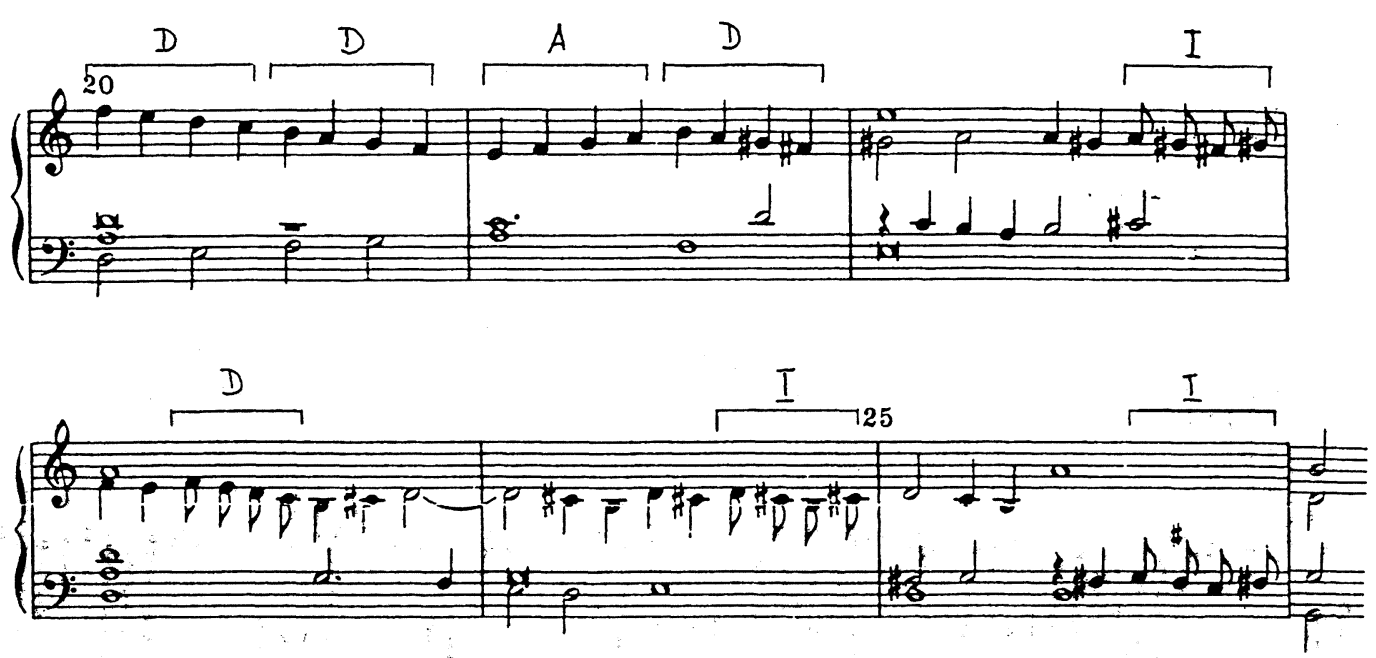

Als feste Formel tritt die viertönige Achtelgruppe auf, die den jeweiligen Zielklang vorbereitet. In den Orgeltraktaten des 15. Jahrhunderts zählt sie zu den Tactus indifferentes (I), die dort neben den Tactus ascendentes (A) und Tactus descendentes (D) angeführt werden. $\mathrm{Zu}$ letzteren gehören im obigen Beispiel die absteigenden Achtel in Takt 23. Als Viertel erscheinen die Ascendentes und Descendentes in Takt 21. Aber auch in dem absteigenden Oktavgang von Takt 20 werden zwei Descendentes aneinandergereiht. Die Formeltypen sind somit voll in musikalischen Verlauf integriert, und geben ihm sein Gepräge, so dass die Reduktion auf einen vermeintlichen Kernsatz unmöglich ist. Was übrig bliebe, wäre ein Torso von Einzelklängen, die nur harmonisch aufeinander beziehbar sind. Erst durch die sinnvolle Plazierung der Spielformeln, die vor allem die Taktanfänge anzielen und von Klang zu Klang einen Bogen schlagen, entsteht ein tatsächliches Orgelstück. Das taktweise Vorgehen beruht auf einem bewährten Prinzip, das schon im 15. Jahrhundert das Tastenspiel kennzeichnet und bis in die Gegenwart weiterwirkt. Der Takt fungiert dabei nicht nur quantitativ zeitabsteckend, sondern besitzt ausserdem eine musikalisch-inhaltliche Komponente. ${ }^{8}$

6. Michael Kugler, Die Musik für Tasteninstrumente im 15. und 16. Jahrhundert, Wilhelmshaven 1975.

7. Knud Jeppesen, Die italienische Orgelmusik am Anfang des Cinquecento, Bd.II, Oslo usw., 2. Auflage 1960, S. 10, T. $20-26$.

8. C. Bockmaier, s. Anm. 4. 
Das Spezifische des Tastenspiels zeigt sich auch in den Intabulierungen vokaler Kompositionen, wie den "Frottole intabulate da sonare organi". Dem bei Andrea Antico erschienenen ältesten Druck von Orgelmusik (Rom 1517) ist der folgende intabulierte Abschnitts der Frottole "Non piu morte morire" von Bartolomeo Tromboncino entnommen:"

Beispiel 2:

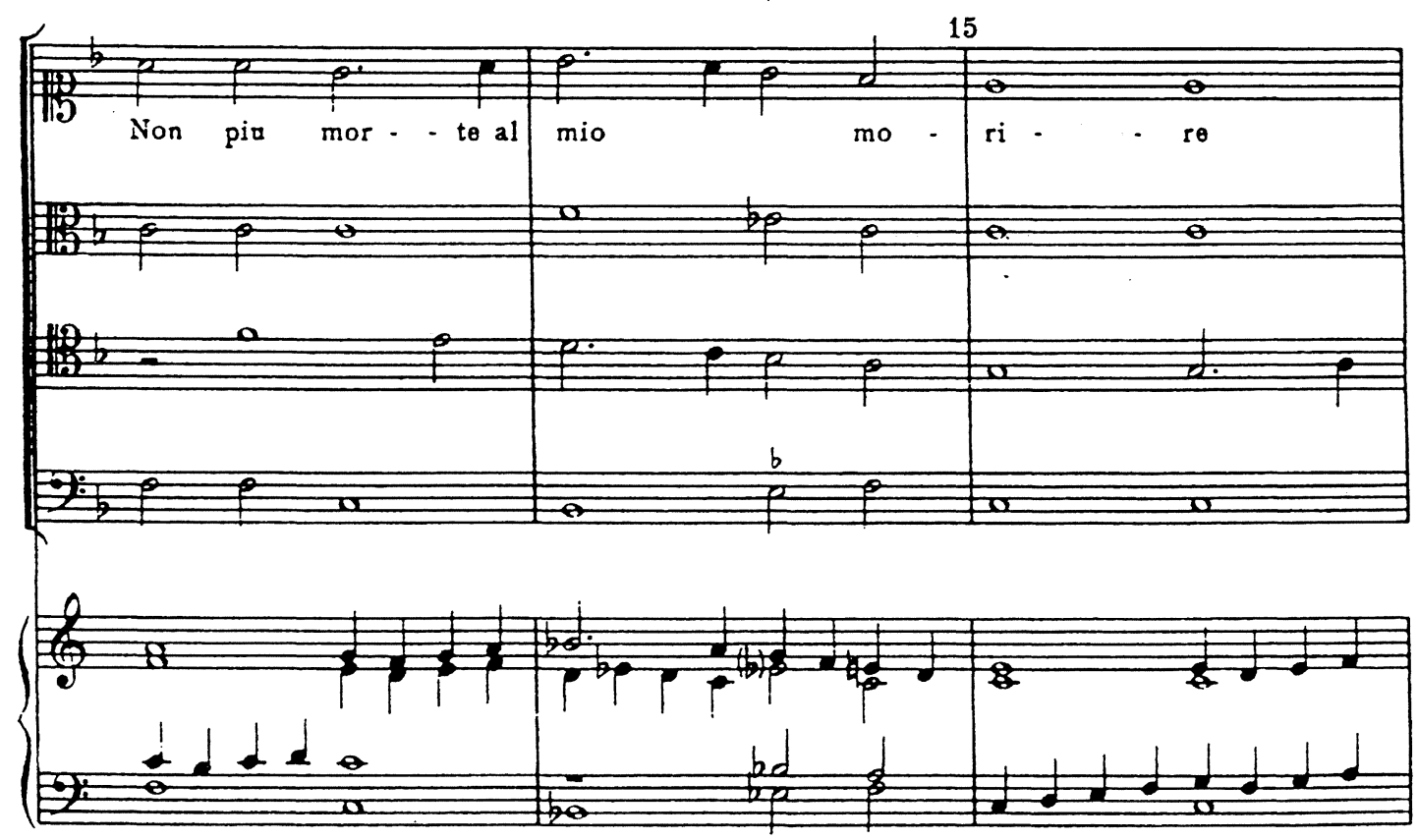

Hier verwandelt sich der rhythmisch bewegte Vers (Ottonario) in einen uniformen Spielvorgang, der sich von Takt zu Takt mit seiner mechanischen Reihung von Viertonformeln wiederholt. Dabei werden aus dem Stimmenverband der Vorlage die Klänge herausgelöst und zu Stützen von Spielformeln. Wieder ist es der Takt, der auf der instrumentalen Ebene erscheint und den Bewegungsfluss gliedert. Der Vorgang unterscheidet sich nur wenig von dem Bewegungskontinuum in Cavazzonis Ricercar. Hier wie dort prägen die Formeln das Spiel, obwohl der Ausgangspunkt ein anderer ist. Auch die Intabulierung begnügt sich nicht mit einer notengetreuen Übernahme der Vorlage, sondern behauptet sich in ihrer instrumentalen Autonomie.

Eine spezielle Verbindung von originalnaher Intabulierung und instrumentaler Selbständigkeit gehen die "Fabordones con glosas" Antonio de Cabezóns ein. ${ }^{10}$ In Anlehnung an die mehrstimmige Psalmrezitation (Falsobordone) wird ein vierstimmiger Vokalsatz auf das

9. K. Jeppesen, Anm. 7, Bd. I, Anhang S. 19, T. 13-15.

10. Antonio de Cabezón, Obras de música para tecla, arpa y viuela, Madrid 1578; Monumentos de la música espanola, hrsg. von. Higinio Anglés, Barcelona 1966, Instituto español de musicología, Vol. XXVII, S. 48-49. 
Tasteninstrument übertragen und dient als "Thema" für Variationen (Glosas). Während letztere das Instrumentalspiel mit dem gängigen Formelmaterial enthalten, hat das Thema vokalen Charakter, obwohl es nicht mit der psalmodischen Vorlage identisch ist. Im Unterschied zum vokalen Falsobordone, der zwischen freier Sprachrezitation und rhythmisch fixierten Kadenzen wechselt, ist bei Cabezón das Fabordon-Thema durchgehend rhythmisiert und in Takten notiert. Es handelt sich also um eine besondere Art von Intabulierung, die die Vorlage in einigen charakteristischen Zügen modifiziert und für den instrumentalen Gebrauch einrichtet: ${ }^{11}$

\section{Beispiel 3:}

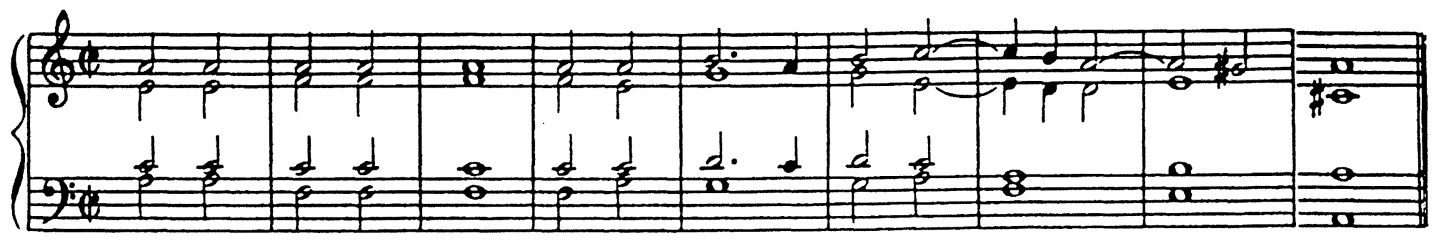

Man kann die folgenden Glosas, in denen zuerst die Oberstimme, dann der Bass und abschließend die zwei Mittelstimmen variiert werden, sinngemäß als instrumentale Kommentare zu dem sprachgebundenen Thema verstehen. Als Intabulierungen zeichnen sie sich im Vergleich zu dem Antico-Beispiel nicht zuletzt durch die didaktische Absicht aus, die Anordnung der Spielformeln nach Stimmen getrennt vorzunehmen. An dem grundsätzlichen Verfahren der zielgerichteten Taktausfüllung ändert sich aber nichts: ${ }^{12}$

\section{Beispiel 4:}
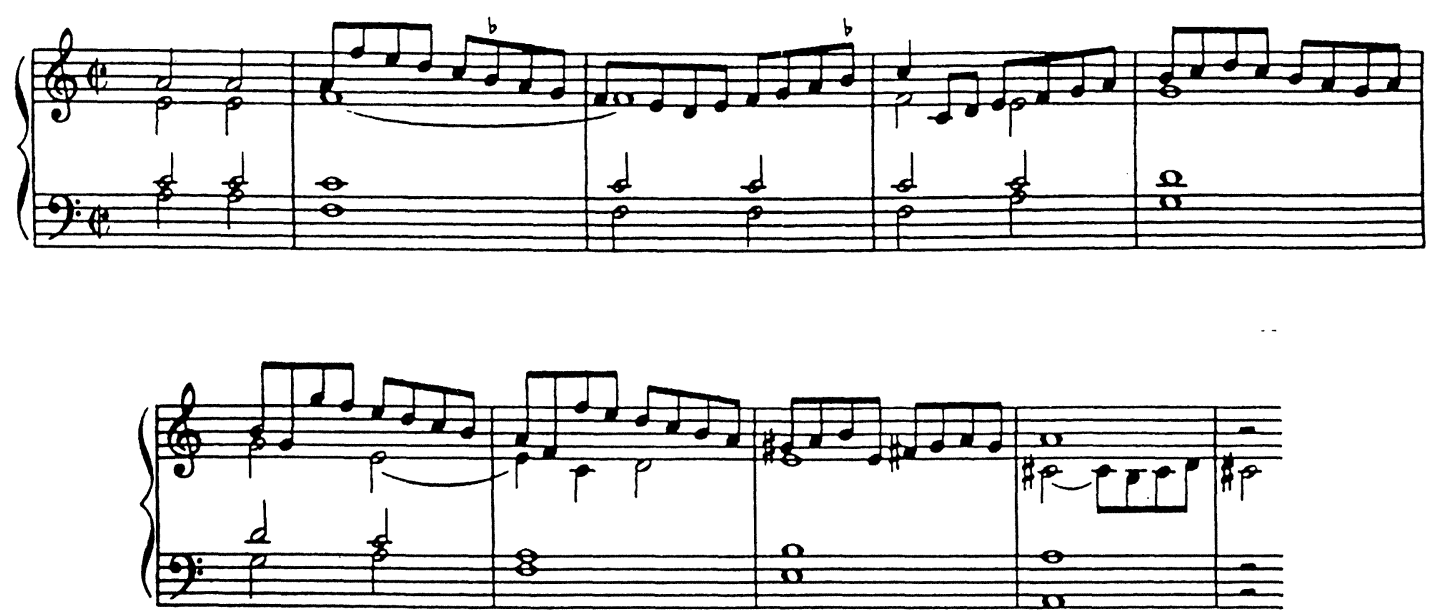

11. Cabezón, Anm. 10, S. 48. "Fabordones del primer tono", 1. Halbvers.

12. Cabezón, Anm. 10, S. 49. "Glosado en el tiple", 1. Halbvers. 
In den Glosas Cabezóns, die zu den frühesten Psalm-Versetten zählen, begegnen wir einer neuen tasteninstrumentalen Gattung: der Variation. Die sich stets ändernde Verteilung der Formeln intensiviert die instrumentalen Möglichkeiten und macht das Spiel zum Selbstzweck. Es kommt umsomehr zu sich selbst, je weiter es sich vom vokalen Ausgangspunkt entfernt.

Ähnlich wie Cabezón hält noch Girolamo Diruta im "Il transilvano" an der Eigenständigkeit der Spielformel fest. ${ }^{13}$ Während er sonst die Orgelmusik in die Kontrapunktlehre einbezieht und den Anschluß an aktuelle Zeitströmungen findet, ist die Spielformel nach wie vor ein fester Baustein, der nicht weiter unterteilbar und deshalb kontrapunktisch nicht verfügbar ist. Ganz in der Art von Cabezóns Glosas sind Dirutas MinutaBeispiele, in denen die stereotypen Viertonformeln in den intabulierten Stimmen Sopran, Bass, Tenor, Alt erscheinen. Hier der "Soggetto" und die "Minuta sopra la parte del soprano" mit der anschließenden Variante "alio modo" in halbierten Notenwerten: ${ }^{14}$

\section{Beipsiel 5:}

Sogetto

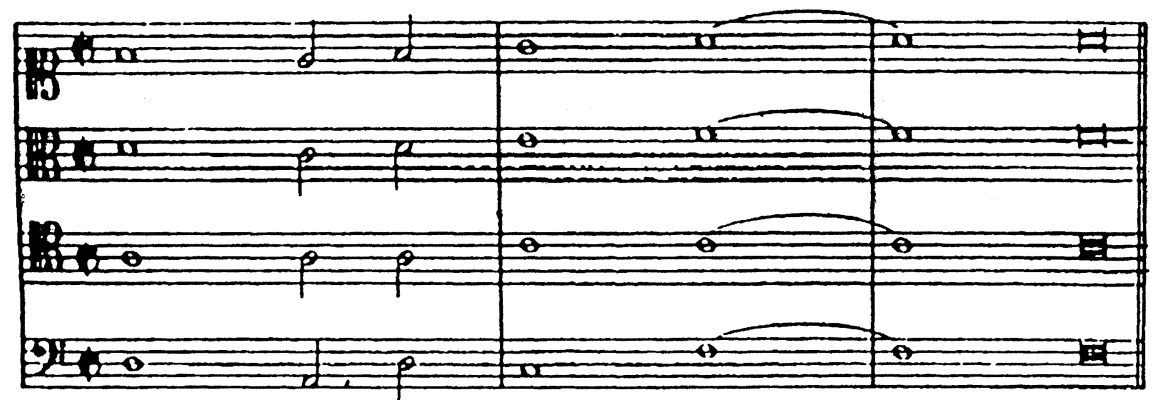

Minuta sopra la parte del Sopriaino
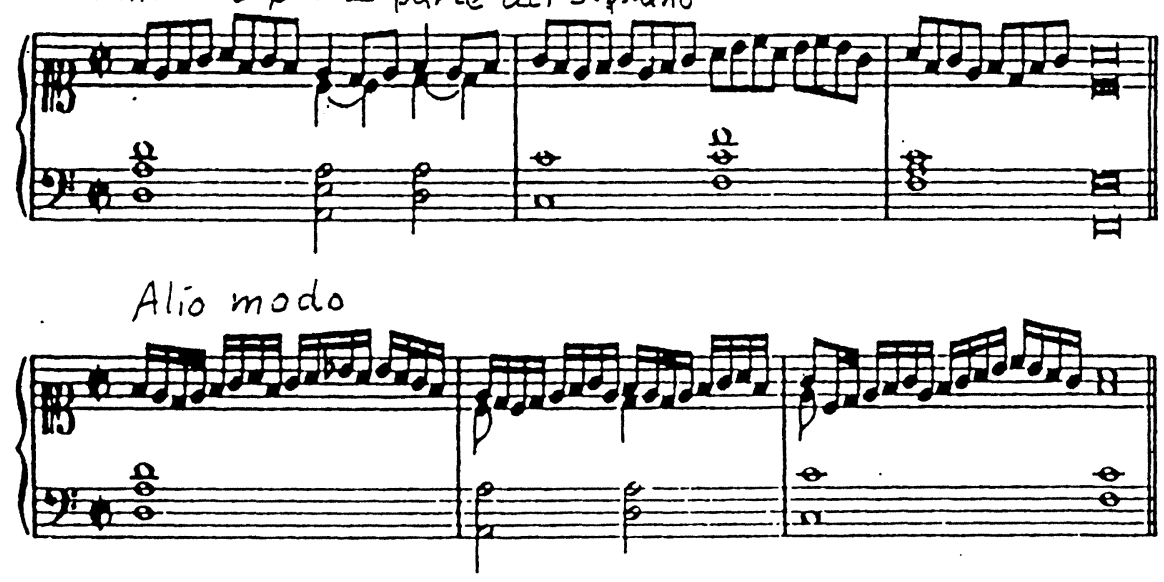

13. Girolamo Diruta, Il transilvano, dialogo sopra il vero modo di sonar organi, et istromento da pena, Venetia 1593; Seconda parte, Venetia 1603. Facsimile, hrsg. von Luisa Corvelli, Bologna 1969.

14. Anm. 13; Seconda parte, Libro I, S. 11. Carl Krebs, "Girolamo Diruta's Transilvano", in: Vierteljahrsschrift für Musikwissenschaft, VIII, 1892, S. 350. 
Dass Diruta von der Formel und nicht vom Einzelton ausgeht zeigt sich auch an der Bezeichnung "Minuta". Bei anderen formelhaften Gebilden spricht er von "Groppo", "Tremolo", "Clamatione", "Accento" und hält sich an das auch sonst üblische Vokabular. Hierin wirkt zweifellos der alte Organistenbrauch nach, stereotype Wendungen mit Namen zu versehen und zu klassifizieren. ${ }^{15}$

Der Begriff Minuta jedoch weist noch in eine andere Richtung, nämlich auf die Diminutio, von der schon im Untertitel des "Transilvano" die Rede ist und worum es auch im Minuta-Abschnitt geht (L`intavolar diminutio). Obwohl der Sache nach mit den Spielformeln der Organisten identisch, sind Blickrichtung und terminologische Herkunft anderen Ursprungs. Denn der Begriff "Diminutio" entstammt der vokalen Kontrapunktlehre des 14. und 15. Jahrhunderts und bezieht sich auf die Unterteilung grösserer Notenwerte des Note-gege-Note-Satzes (punctus contra punctum). ${ }^{16}$ Ausgangspunkt ist in der Regel die Brevis, die in verschiedenen Kombinationen von Semibreven und Minimen unterteilt werden kann. Dabei löst sich die syllabische Deklamation in längere Melismen mit oft durchgehenden Minimen auf. Diruta scheint also in der Verwendung von "Diminutio" für das Orgelspiel die vokale Terminologie übernommen zu haben, obwohl es nach wie vor bei den alten Spielformeln bleibt.

Anders steht es dagegen mit Spielanweisungen für Blas- und Saiteninstrumente, die also für Melodieinstrumente bestimmt sind und wie Vokalstimmen behandelt werden. Schon das älteste diesbezügliche Lehrbuch, die "Opera intitulata fontegara" von Sylvestro Ganassi (Venedig 1535) geht ganz selbstverständlich mit dem Verbum "Diminuir" um, das schon im Titel auf den Zweck der Anleitung hinweist. ${ }^{17}$ Nicht nur im Wortgebrauch, sondern auch in Ansatz und Durchführung unterscheidet sich Ganassi von der Organistenpraxis. Hatte Conrad Paumann sein "Fundamentum organisandi" von 1452 mit drei Viertonformeln in einem Tactus begonnen und ging erst bei den abschließenden Klauseln zu Einzeltönen über, so stehen diese bei Ganassi in Form eines einfachen Intervallschritts am Anfang der nachfolgenden Diminutionsbeispiele. ${ }^{18}$ Die darin vorkommenden Viertonformeln sind also nicht von vornherein da, sondern das Ergebnis sukzessiver Verkleinerung des Ausgangstons. Auch beim didaktischen Fortschreiten von wenigen Diminutionstönen bis zu umfangreichen Gebilden, vom Einfachen zum Komplexen, steht die neue Diminutionslehre in der Nachfolge der älteren. Doch geht sie in der einseitigen Fixierung auf spielerische Virtuosität weit darüber hinaus, zumal sie sich weniger auf die kompositorische Faktur, als auf die Erlernung des Instruments bezieht. Beherrschung von Instrument und Diminution gehen Hand in Hand.

15. S. oben S. 3; Th. Göllner, Anm. 4, S. 62, 164, 185

16. Klaus-Jürgen Sachs, "Die Contrapunctus-Lehre im 14. und 15. Jahrhundert", in: Frieder Zaminer (Hrsg.), Geschichte der Musiktheorie, Bd. 5, Darmstadt 1984, S. 236-256. Theodor Göllner,"Diminutio und Tactus", in: Michael Bernhard (Hrsg.), Quellen und Studien zur Musiktheorie des Mittelalters III, Bayerische Akademie der Wissenschaften, Veröffentlichungen der Musikhistorischen Kommission, Bd. 15, München 2001, (in Vorbereitung)

17. Opera Intitulata Fontegara / Laquale insegna a sonare di flauto chon tutta l'arte opportunata a esso instrumento massime il diminuire...Faksimile-Ausgabe: Biblioteca Musica Bononsiensis, Sezione II N. 18, Bologna 1969.

18. Willi Apel (ed.), "Keyboard Music of the Fourteenth and Fifteenth Centuries", in: Corpus of Early Keyboard Music, I, 1963, S. 32. Sylvestro Ganassi, Opera intitulada Fontenagra, Venedig 1535, Hildemarie Peter (ed.), BerlinLichterfelde 1956, S. 40-41. 
$\mathrm{Zu}$ einer neuen Situation kommt es, wenn dasselbe Prinzip vom instrumentalen auf den vokalen Bereich übergreift und die Diminution im Sologesang stattfindet. Die Voraussetzungen hierzu sind keineswegs so selbstverständlich wie im Instrumentalen. War dieses von Anfang an auf die Spielformeln angewiesen, die gleichsam als Identitätsmerkmal fungierten, so gelten für den Gesang andere Gesetze. Auch der Solosänger musste im 16. Jahrhundert zunächst und vor allem einen Text vortragen und somit Sprache vermitteln. Er konnte dann, falls er die Kunst des Diminuierens beherrschte, die Sprachdeklamation aussetzen und musikalische Verzierungen anbringen. Die instrumentale Komponente betrifft aber nur einen Teilbereich des Gesangs und steht im Gegensatz zur artikulierten Sprache. Es ist bekannt, dass vokale Verzierungen zunächst improvisiert wurden, bevor sie im letzten Jahrzehnt des 16. Jahrhunderts in italienischen Gesangsschulen und Beispielsammlungen erscheinen (s. oben S. 2).

Dass es bis dahin im Gegensatz zu den zahlreichen instrumentalen Spielanweisungen keine entsprechenden vokalen Verzierungslehren, sondern nur gelegentliche Hinweise gibt, mag seinen Grund in dem geringeren Bedarf haben. ${ }^{19}$ Während der Organist sein Handwerk ohne Spielformeln garnicht erlernen und ausüben konnte und generell die Instrumentalisten didaktische Anleitungen benötigten, waren die Sänger nicht darauf angewiesen. Für sie genügte es, ob in chorischer oder solistischer Besetzung, die Komposition, so wie sie schriftlich fixiert war, also ohne Verierungen aufzuführen. Mit anderen Worten: Jeder Instrumentalist benötigte seine Spielformeln, aber nicht jeder Sänger musste sich durch Diminutionen und Passaggien hervortun. ${ }^{20}$ Dies dürfte $\mathrm{zu}$ dem verhältnismässig späten Erscheinen vokaler Diminutionslehren beigetragen haben. Bis dahin konnte sich der qualifizierte Sänger an die instrumentalen Diminutionslehren halten, die oft im Titel einen ähnlichen Zusatz aufweisen, wie schon Ganassis "Fontegara"21 : Ad ogni instrumento di fiato et corde, et ancora a chi si dileta di canto". Ähnlich heißt es bei Girolamo Dalla Casa: "Il vero modo di diminuir... di fiato \& corda \& di voce humana" (Venedig 1584). ${ }^{22}$

Erst in Dalla Casas zweitem Lehrwerk von 1590 geht es ausschließlich um die vokale Verzierungskunst. ${ }^{23}$ Wie schon bei den Instrumentallehren werden die Beispiele nach auf- und absteigenden Intervallschritten, Kadenzformeln usw. geordnet, worauf sich Diminutionen konkreter Kompositionen anschließen. Der verzierten Vokalstimme wird der Text unterlegt, der auch für die darüber notierte Originalstimme gilt, wie bei folgendem Ausschnitt aus dem von G.B. Bovicelli diminuierten "Ave verum" Palestrinas: ${ }^{24}$

19. H.M. Brown, Anm. 2.

20. H.M. Brown, Anm. 2, S. 64: "...the theoretical sources seem to indicate that only some singers but all instrumentalists had to be able to embellish their parts."

21. Vgl. auch Anm. 17.

22. Howard Mayer Brown, Instrumental Music Printed before 1600, A Bibliography, Cambridge, Massachusetts 1967 , S. 328

23. Il secondo libro di madrigali a cinque voci, con i passaggi. Venetia 1590. Ernest T. Ferand, Anm. 3, S.157. H.M. Brown, Anm. 2, S. 65. In kurzer Folge erscheinen drei weitere vokale Verzierungslehren: Lodovico Zacconi, Prattica di musica (Venedig 1592) Cap.66. Giovanni Luca Conforti, Breve et facile maniera...a far passaggi (Rom 1593). G.B. Bovicelli, Regole Passaggi...(s. Anm. 24).

24. Giovanni Battista Bovicelli, Regole Passaggi di musica. Madrigali e motetti passaggiati, Venetia 1594. Faksimile-Ausgabe von Nanie Bridgman, Documenta Musicologica XII, Kassel und Basel 1957, S. 44. Teil-Edition bei Hugo Goldschmidt, Anm. 1, Anhang S. 24-25. 
Beispiel 6:

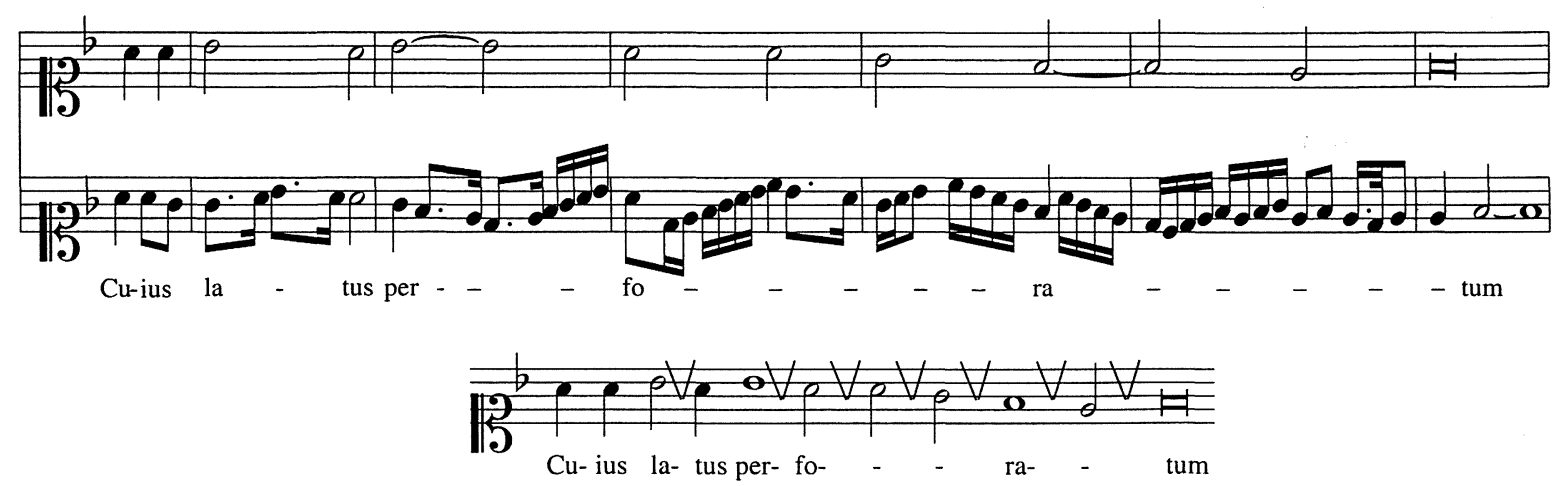

Die zwei Versionen werden durch Vertikalstriche koordiniert, die die Zuordnung der gehäuften kurzen Noten erleichtern und zugleich als Taktstriche im Abstand von Semibreven gelten. Ausserdem gliedern sie die Diminution in sinnvolle Einheiten. Dies wird schon im zweiten Takt deutlich, wo auf der Silbe "per-" die Bewegung zunächst zur Taktmitte hin in die Unterquart fällt, um dann im raschen Aufstieg den nächsten Taktbeginn $(a)$ anzusteuern. Der neue Takt auf der Silbe "fo-" und dem wiederholten $a$ bringt einen weiteren Bewegungsimpuls mit emporschnellenden Sechzehntteln zur Taktmitte hin. Auch der vierte Takt hat sein eigenes Profil: auf- und absteigende Sechzehntel, unterbrochen durch ein Achtel, Viertel auf der Taktmitte und Zielformel zum nächsten Takt. Sodann ein nochmaliger Diminutionstakt: kreisende Sechzehntel, Achtel auf Kernton und Taktmitte, Zielformel mit punktierter Betonung und vorhaltiger Einsatz des Schlusstons auf der Endsilbe "-tum".

Um eine vorgegebene Singstimme $\mathrm{zu}$ verzieren, ändert die Diminution deren Physiognomie durch Vorgänge, die dem melodischen und sprachlichen Zusammenhang entgegenstehen. Die völlig verfremdete Ausgangsstimme mit dem Text "cuius latus perforatum" gelangt in ihrer Originalform nach zwei auftaktigen Vierteln und dem wiederholten $b$ in absteigenden Sekundschritten von $b$ nach $f$, wobei die syllabische Deklamation kaum verlassen wird. ${ }^{25}$ Im Vergleich dazu lässt die diminuierte Fassung (Beispiel 6) gerade noch die Anfangsworte erkennen. Dann aber schieben sich die Passaggien wie Keile zwischen Töne und Silben, trennen sie, um sie auf andere Weise wieder zu verbinden. Es tritt eine neue Ordnung ein, in der der Takt eine wichtiges Mittel ist, um den Bewegungsfluss zu steuern.

Das Aufbrechen eines sprachlich-musikalischen Sinnzusammenhangs, das die italienische Verzierungskunst des späten 16. Jahrhunderts kennzeichnet, ist an sich nicht neu. Der solistische Gesang kennt von jeher ausgedehnte melismatische Partien, die in der liturgischen Ein- und Mehrstimmigkeit ebenso wie in der weltlichen Musik die Sprachdeklamation zurücktreten lassen. Neu ist aber ihre enge Verflechtung mit dem gleichzeitigen

25. Palestrinas "Ave verum" ist die Kontrafaktur seines Madrigals "Io son ferito", das gleichfalls von Bovicelli diminuiert wurde und dem "Ave verum" vorausgeht (S. 38-42). Die entsprechende Stelle "Nè getta sangue la mia piaga nuova" ist im Original streng syllabisch. 
Instrumentalspiel. Es sind vielfach dieselben Formeln, die hier wie dort auftreten. Auch in den Lehrbüchern wird dieselbe didaktische Methode in der Ausgestaltung von Intervallen angewandt, die von einfachen zu komplizierten Beispielen fortschreitet und schliesslich ganze Kompositionen einbezieht.

Angesichts dieser Gemeinsamkeiten stellt sich die Frage nach der genetischen Abhängigkeit. Die Autoren der instrumentalen Diminutionslehren geben den Rat, der menschlichen Stimme nachzueifern. So beginnt der Flötist Sylvestro Ganassi seinen Traktat mit dem Satz: "Voi havete a sapere come tutti li instrumenti musicali sono rispetto et comparatione ala voce humana."26 Diese Reverenz vor der menschlichen Stimme will jedoch weniger der vokalen Diminution den Vorrang geben als vielmehr die hohe Kunst der an den Sprachvortrag gebundenen Stimme betonen, die dem instrumentalen Handwerk übergeordnet ist. In der Praxis des Diminuierens dagegen steht das Instrument im Vordergrund, das unabdingbar auf die Spielformel angewiesen ist, und die menschliche Stimme nimmt einen nachgeordneten Platz ein, worauf schon in den Buchtiteln hingewiesen wird. ${ }^{27}$

Die Abhängigkeit vom Instrumentalen geht so weit, dass auch das Variationsprinzip wie in den "Glosas" Cabezóns oder den "Minuta" Dirutas von den vokalen Diminutionslehren und Beispielsammlungen übernommen wird. Ähnlich wie bei Cabezón wird in den vokalen Falsibordoni zunächst das "Thema" ohne Diminution vorgestellt, bevor die instrumentale Arbeit des Variierens beginnt. Nicht selten steigert sich von Vers zu Vers der Diminutionsgrad, der in der abschliessenden Doxologie seinen Höhepunkt erreicht. In den "Salmi passaggiati" von Frencesco Severi (Rom 1615) ${ }^{28}$ wechseln, ähnlich wie in den instrumentalen Vorläufern, einzelne Stimmen (Canto, Alto, Tenore, Basso) versweise, wobei lediglich die Reihenfolge offen bleibt. Der Weg vom mehrstimmig-chorischen zum solistisch-diminuierten Psalmvortrag scheint also auch über den Umweg der instrumentalen Intabulierung zu verlaufen oder wurde zumindest von dorther angeregt. Auf jeden Fall ist diese wesentlich früher belegt als ihr vokales Gegenstück. Es bleibt allerdings ein gravierender Unterschied, der für die vokale Psalmodie schlechthin gilt: Auch die Diminution kann die sprachliche Rezitation nicht völlig aufheben, so dass das instrumentale Prinzip fortwährend unterbrochen wird. Die syllabisch rezitierenden Abschnitte stellen der instrumentalisierten Stimme einen Widerstand entgegen, der auf sprachlicher Artikulation und formaler Syntax beruht. In Severis passaggierten Psalmen stehen rhythmisch frei rezitierte Abschnitte neben diminuierten, in Takten notierten Partien. Aber auch, wenn die Rezitation rhythmisch exakt in Takten fixiert ist, wie in den "Salmi passaggiati" von G.L. Conforti (Rom 1602) ${ }^{29}$, tritt dieser Gegensatz unverändert auf: $:^{30}$

26. S. Ganassi, Fontegara, Anm. 17. Cap. I "Dechiaration del suo termine".

27. s. Anm. 22.

28. Salmi passaggiati per tutte le voci nella maniera che si cantano in Roma...(Roma 1615). Murray C. Bradshaw (ed.), Francesco Severi, Salmi Passaggiati (1615), Madison, Wisconsin 1981. Theodor Göllner. "Nos qui vivimus: Stages of a Polyphonic Psalmverse"; in: The Echo of Music. Essays in Honor of Marie Louise Göllner, in Vorbereitung.

29. Tenore, Salmi passaggiati sopra tutti $i$ toni.(Roma 1602). Murray C. Bradshaw (ed.), Giovanni Luca Conforti "Salmi Passaggiati (1601-1603), Neuhausen-Stuttgart 1985. 125, T. $48-67$.

30. Palm 111 "Beatus vir", Vers 5, Severi, Bradshaw (ed.), S. 15-16, T. 24-27. Conforti, Bradshaw (Ed.), S.124- 
Beispiel 7:

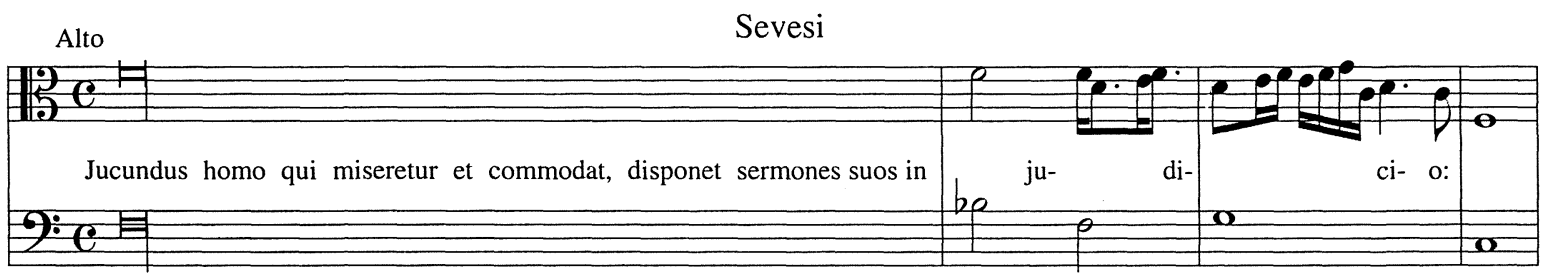

Beispiel 8:
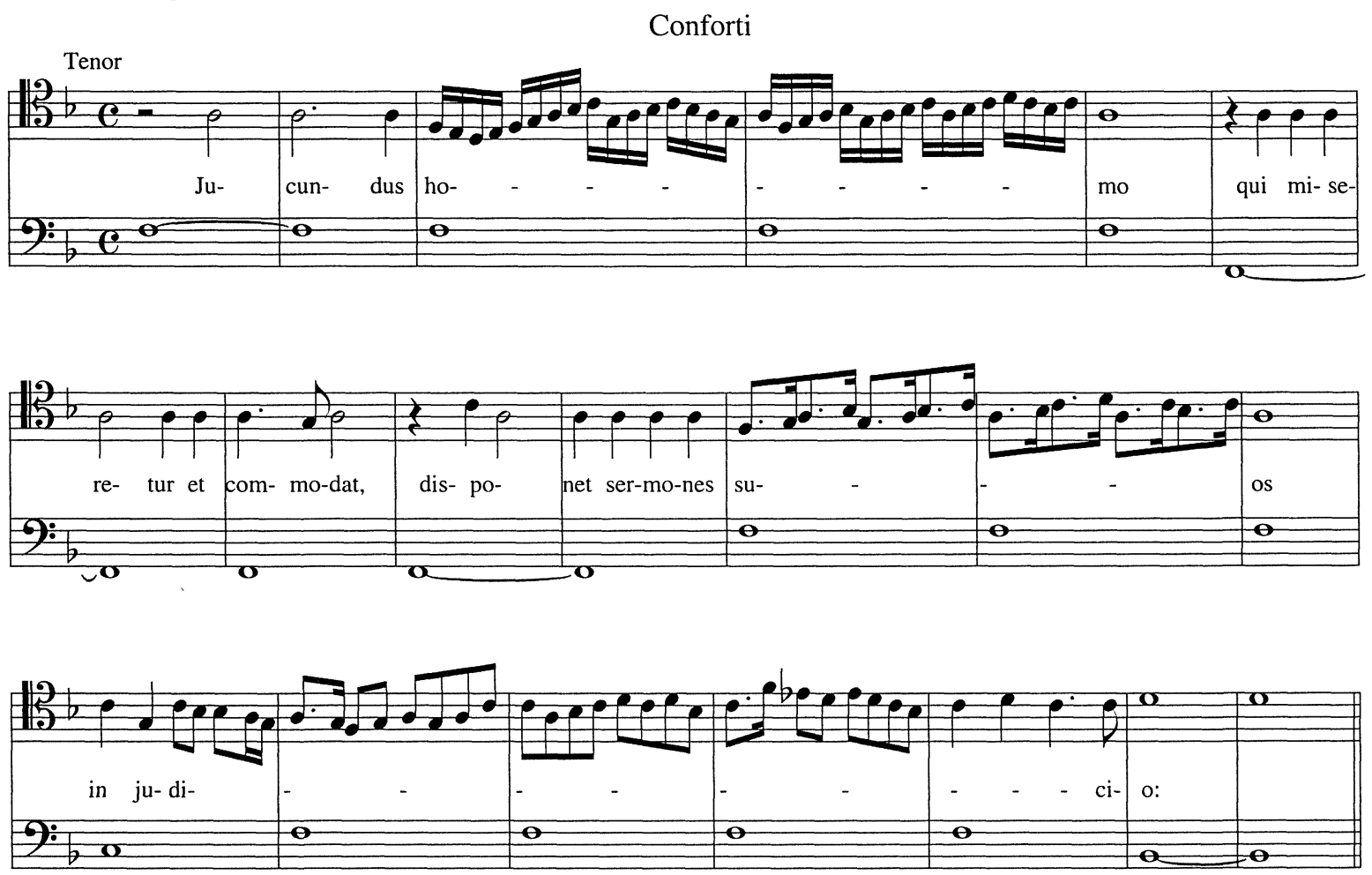

In den beiden Ausschnitten ist die Verteilung von rezitierten und verzierten Partien uneinheitlich. Während Severi den Text durchgehend rezitiert und nur die Mediatio verziert, gliedert Conforti nach kleineren syntaktischen Einheiten und fügt dreimal längere Diminutionen auf Akzentsilben hinzu. Im Vergleich zu Severi, der ganz dem Falsobordone-Schema folgt, geht Conforti differenzierter vor und nähert sich rezitativischer Deklamation. In beiden Fällen wird jedoch mit syllabischen Partien begonnen, bevor die Diminution auf der letzten Akzentsilbe einsetzt. Es ist dies nichts weiter als die modernisierte Form der traditionellen Falsobordone- 
Klausel, die sich gleichfalls von der Rezitationstuba löst. Conforti bringt im Gegensatz zu Severi Passaggien auch zur Binnengliederung an und verleiht andererseits der Sprachdeklamation durch Einbeziehung in die Taktmensur grösseres Gewicht. Was dem Sprachverlauf durch wiederholt eingefügte Passaggien verlorengeht wird gleichsam durch die rhythmisch gefestigte Syllabik kompensiert. Aber weder die Rezitation der Sprache noch deren Verschwinden in umfangreichen Diminutionen berühren die Bedeutungsebene. Die mit Melismen versehenen Abschnitte signalisieren Zäsuren, aber keine Wortinhalte. ${ }^{31}$

Hatte Caccini in seiner programmatischen Vorrede zu "Le nuove musiche" die Kunst des Passaggierens einschränken wollen, da sie sein wichtiges Ziel, die Musik der gesprochenen Rede anzugleichen, gefährdet, so war die musikalische Praxis noch weit entfernt davon. Conforti und Severi verdanken ihren Ruhm als gefeierte päpstlichen Capellsänger allein ihren virtuosen Gesangsleistungen. Was sie an Sprache mitteilen, steht ganz im Schatten des glanzvollen Passaggierens. Bei den Psalmen wiederholt sich von Vers zu Vers derselbe Vorgang. Der rezitierte Text ist gleichsam nur die Vorbereitung, der Anlauf zu den kulminierenden Diminutionen, die Zäsuren und Schlüsse herbeiführen. Die Stimme zeigt sich von ihrer instrumentalen Seite, und in der Tat geht der Weg dorthin vom Instrumentalspiel aus, das jetzt einen faszinierenden Platz im Vokalen erhalten hat.

31. Eine andere Meinung vertritt Murray Bradshaw im Hinblick auf Conforti, bei dem er ausdruckserfüllte Passagen ("expressions") nachzuweisen versucht. Murray C. Bradshaw, "Text and Tonality in Early Sacred Monody"; in: Musica Disciplina XLVII, 1993, S. 170-225, hier S. 194-203. Ders., "Giovanni Luca Conforti and Vocal Embellishment: From Formula to Artful Improvisation"; in: Performance Practice Review, Vol.8, Nr.1, 1995, S. 5-27. 